\title{
Application of building simulation tools for studying airborne infection and its control
}

๑) Tsinghua University Press and Springer-Verlag Berlin Heidelberg 2012

Following the 2003 SARS epidemic, 2009 influenza pandemic and ongoing human avian influenza infections, there has been growing concern of the potential of airborne transmission of infection. Exposure of infectious droplets or droplet nuclei within a short range or long range from the source can lead to infection, though the relative importance of such airborne infection for some diseases such as influenza is unknown. Various well developed engineering tools such as building simulation methods have been applied to study the generation and transport of the expiratory droplets, the impact of using building ventilation and personalized ventilation for infection control, evaporation and dispersion of respiratory droplets, the effectiveness of some personal protective equipment such as masks, and design of negative isolation rooms. These engineering tools are not only useful to study the effectiveness of various infection control methods, but also useful to help studying the airborne transmission mechanisms and designing airborne pathogen samplers. Early studies also demonstrated the usefulness of these tools for investigating the possible airborne outbreaks (Yu et al. 2004).

Most often, these building simulation tools are used together with experimental methods such as flow visualization and airflow measurement. The argument for such a combined approach is simple as the two methods can complement each other ( $\mathrm{Li}$ and Nielsen 2011). Experimental methods may suffer from uncertainties due to inherent scaling errors. By scaling errors, we mean that an experiment cannot always reproduce the exact physical scales and settings. Simulation methods may also suffer from uncertainties due to inaccurate mathematical models such as turbulence modeling, boundary conditions and the appropriate numerical solution methods. Both simulation and experimental tools for studying airborne infection are recently reviewed in Tang et al. (2011).

Application of building simulation tools in airborne infection studies is somewhat similar to other applications such as diffusion and control of chemical pollutants, control of PM2.5 and PM10, and thermal transport and thermal comfort in buildings. This may explain how building simulation professionals have been able to quickly found themselves indispensable to the airborne infection control community after the SARS epidemics. We have seen leading microbiologists, epidemiologists and infection control professionals have teamed up with computational fluid dynamists and ventilation experts to understand the airborne transmission mechanisms, while engineers teaming up with the medical professionals in developing effective engineering control methods for infection control in critical environments such as schools, hospitals and aircraft cabins. However, we all know too well that such a multi-disciplinary effort is not an easy task, though it is a right direction.

Airborne infection studies have their own characteristics. For example, the fact that expiratory droplets are the vectors for respiratory infection suggests the importance of studying release and capture of these droplets or their drying up product (droplet nuclei) at mouth and/or nose. The vortex dynamics and the near body micro-environment are all critical to this release and capture process. Hence a thermal breathing manikin with much more details than those commonly used in building ventilation study is mostly needed in airborne infection studies.

The 7 articles presented in this Topical Issue cover a range of topics of airborne infection and control, including the back tracking the location of index patient in a SARS outbreak, the effectiveness of sliding doors on operating theatre environment, the roles of ventilation rates, intervention strategies in schools, negative pressure control, the locking-up phenomenon of droplets in displacement ventilation, etc. The building simulation tools include not only the conventional computational fluid dynamics, but also a probability-based inverse modeling method, and a social indoor contact model integrated with the Wells-Riley equation.

Chinese philosopher Confucius had once said, "The mechanic that would perfect his work must first sharpen his tools (工欲善其事, 必先利其器)”. Building simulation tools are essential in studying airborne infection and control 
as transmission of the airborne diseases mostly occur in buildings. Both innovative application of existing tools and development of new innovative building simulation tools are needed for airborne infection studies, before human being manage to eradicate the common cold, seasonal influenza and other diseases.

We hope that you find this topical collection of the papers useful.

\section{Guest Editors:}

Yuguo Li

Department of Mechanical Engineering

The University of Hong Kong

Hong Kong, China

E-mail: liyg@hku.hk

\section{Bin Zhao}

Department of Building Science

School of Architecture

Tsinghua University

Beijing 100084, China

E-mail: binzhao@tsinghua.edu.cn

\section{References}

Tang JW, Noakes CJ, Nielsen PV, Eames I, Nicolle A, Li Y, Settles GS (2011). Observing and quantifying airflows in the infection control of aerosol- and airborne-transmitted diseases: An overview of approaches. Journal of Hospital Infection, 77: 213 - 222.

Yu ITS, Li Y, Wong TW, Tam W, Chan A, Lee JHW, Leung DYC, Ho $\mathrm{T}$ (2004). Evidence of airborne transmission of the severe acute respiratory syndrome virus. New England Journal of Medicine, 350: 1731 - 1739 .

Li Y, Nielsen PV (2011). CFD and ventilation research. Indoor Air, 21: $442-453$. 